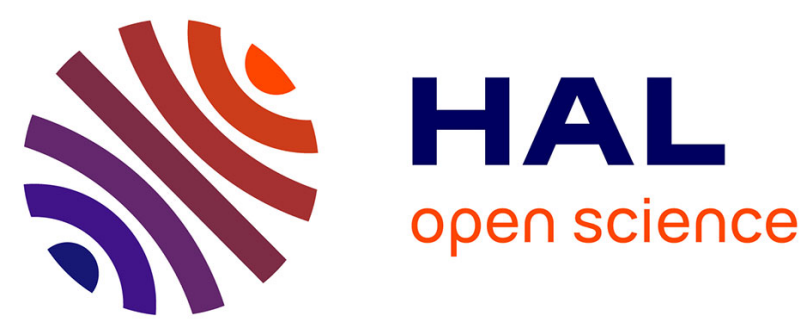

\title{
Emergence of cytotoxic resistance in cancer cell populations: single-cell mechanisms and population-level consequences
}

\author{
Tommaso Lorenzi, Rebecca H. Chisholm, Alexander Lorz, Annette K Larsen, \\ Luís Almeida, Alexandre Escargueil, Jean Clairambault
}

\section{To cite this version:}

Tommaso Lorenzi, Rebecca H. Chisholm, Alexander Lorz, Annette K Larsen, Luís Almeida, et al.. Emergence of cytotoxic resistance in cancer cell populations: single-cell mechanisms and populationlevel consequences. ICNAAM 2015 Session 70: "Mathematical models and methods to investigate heterogeneity in cell and cell population biology", Jean Clairambault, Sep 2015, Rhodes, Greece. hal-01249239

\section{HAL Id: hal-01249239 \\ https://hal.inria.fr/hal-01249239}

Submitted on 4 Jan 2016

HAL is a multi-disciplinary open access archive for the deposit and dissemination of scientific research documents, whether they are published or not. The documents may come from teaching and research institutions in France or abroad, or from public or private research centers.
L'archive ouverte pluridisciplinaire HAL, est destinée au dépôt et à la diffusion de documents scientifiques de niveau recherche, publiés ou non, émanant des établissements d'enseignement et de recherche français ou étrangers, des laboratoires publics ou privés. 


\title{
Emergence of cytotoxic resistance in cancer cell populations: single-cell mechanisms and population-level consequences ${ }^{1}$
}

\author{
Tommaso Lorenzi*, Rebecca H. Chisholm ${ }^{\dagger}$, Alexander Lorz**, Annette K. Larsen ${ }^{*}$, \\ Luís Neves de Almeida ${ }^{* *}$, Alexandre Escargueil ${ }^{*}$ and Jean Clairambault ${ }^{* *}$ \\ ${ }^{*}$ Centre de Mathématiques et de Leurs Applications, ENS Cachan, CNRS, Cachan 94230 Cedex, France \& \\ INRIA-Paris-Rocquencourt, MAMBA Team, Domaine de Voluceau, BP105, 78153 Le Chesnay Cedex, France \\ ${ }^{\dagger}$ School of Biotechnology and Biomolecular Sciences, University of New South Wales, Sydney NSW 2052, \\ Australia \\ ${ }^{* *}$ Sorbonne Universités, UPMC Univ Paris 06, UMR 7598, Laboratoire Jacques-Louis Lions, F-75005, Paris, \\ France \& CNRS, UMR 7598, Laboratoire Jacques-Louis Lions, F-75005, Paris, France \& \\ INRIA-Paris-Rocquencourt, MAMBA Team, Domaine de Voluceau, BP105, 78153 Le Chesnay Cedex, France \\ ¥Sorbonne Universités, UPMC Univ Paris 06, F-75005, Paris, France \& INSERM, UMR_S 938, Laboratory of \\ "Cancer Biology and Therapeutics", F-75012, Paris, France
}

\begin{abstract}
We formulate an individual-based model and a population model of phenotypic evolution, under cytotoxic drugs, in a cancer cell population structured by the expression levels of survival-potential and proliferation-potential. We apply these models to a recently studied experimental system. Our results suggest that mechanisms based on fundamental laws of biology can reversibly push an actively-proliferating, and drug-sensitive, cell population to transition into a weakly-proliferative and drug-tolerant state, which will eventually facilitate the emergence of more potent, proliferating and drug-tolerant cells.
\end{abstract}

Keywords: Cancer modeling; reversible drug tolerance; phenotype evolution; non-genetic instability; stress-induced adaptation PACS: 87.17.Aa, 87.17.Ee

\section{MOTIVATIONS}

In recent experiments, performed on genetically homogeneous populations of cancer cells, Sharma and co-workers [1] showed that epigenetically regulated changes in phenotype can play an important role in the development of reversible drug tolerance. During these experiments, a small subpopulation of drug-tolerant cells was consistently detected, that could maintain viability in the presence of high-dose drug therapy. These Drug-Tolerant Persisters (DTPs) were shown to be non-proliferative and display markers specific to stem cell-like cancer cells. After a period of time, approximately 20 percent of DTPs changed their phenotype to resume normal proliferation and lost stem-like markers, still in the constant presence of the drugs. The resulting cells were labeled the Drug-Tolerant Expanded Persisters (DTEPs). Interestingly, both DTPs and DTEPs could be drug resensitized by drug-free passaging.

Therefore, the three distinct subpopulations - the parental cancer cells (PC9s), DTPs and DTEPs-that compose the whole cancer cell population at various times during drug treatment, although genetically identical, possess different functional phenotypes. Most notably, they can be characterized by their respective levels of survival potential (i.e., the level of robustness towards life-threatening events in extreme conditions, which in this case can be identified as the level of drug tolerance) and proliferation potential (i.e., the rate of cell proliferation) [2]. But what is driving the evolution of phenotypes observed in the PC9 cancer cells? Is it simply a case of selection, where cells with certain properties survive and proliferate better in a given environment [3]? Are individual cells changing their properties in response to environmental cues $[4,5]$ ?

In order to address these questions, in [6] we proposed an Individual-Based (I-B) computational model [4, 7, 8, 9] and an Integro-Differential Equation (IDE) model [10, 11, 12] of the phenotype evolution observed in [1]. Such models can be used as in silico laboratories to test verbal hypotheses, and uncover mechanisms that underlie emergent features of cancer cell populations. The I-B computational model allows an intuitive and flexible description of the system at hand, while the IDE model makes it possible to study the system in terms of qualitative and asymptotic analysis, and

\footnotetext{
${ }^{1}$ T. Lorenzi and R.H. Chisholm contributed equally to this project
} 
(a) Each cell $i$ undergoes either proliferation, death or remains quiescent:

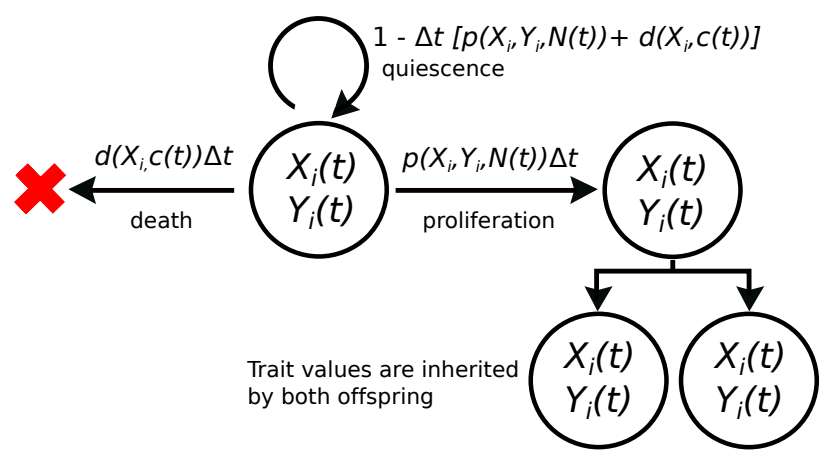

(b) Each cell $i$ updates its trait values according to the discretised SDEs:

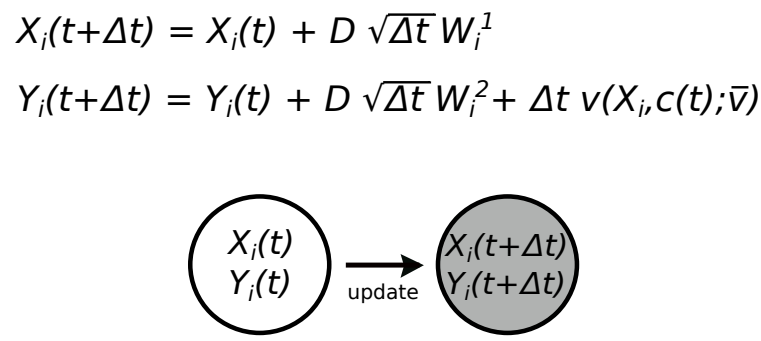

FIGURE 1. Algorithm of the I-B model. (a) In the I-B model, over the interval between two successive time instants $t$ and $t+\Delta t$, each cell $i$ will first either choose to proliferate, undergo apoptosis, or remain in a quiescent state with probabilities $p\left(X_{i}, Y_{i}, N(t)\right) \Delta t, d\left(X_{i}, c(t)\right) \Delta t$ and $1-\Delta t\left[p\left(X_{i}, Y_{i}, N(t)\right)+d\left(X_{i}, c(t)\right)\right]$, respectively. After a cell has undergone one iteration of the proliferation and death process, it will to return to a quiescent state. (b) Secondly, each surviving cell will update its phenotype according to Eqs. (2).

is computationally less expensive.

\section{MATHEMATICAL MODELS}

We describe the evolution of phenotype in a well-mixed PC9 cancer cell population exposed to cytotoxic drugs using both an I-B and an IDE formalism. The concentration of cytotoxic drugs at time $t$ is modeled by the function $c(t)$, and we characterize the state of each PC9 cell by its expression levels of two phenotypic traits: survival potential and proliferation potential. In this framework, we identify a PC9 cell as having a low value of survival potential and a high value of proliferation potential, a DTP cell as having a high value of survival potential and a low value of proliferation potential, and a DTEP cell as having a high value of survival and proliferation potential.

We model the key biological mechanisms of phenotype evolution in the PC9 cancer cells, namely selection, stressinduced adaptation and non-genetic phenotype instability, by using three separate mathematical strategies, which are tailored to fit the I-B and the IDE formalisms. Selection is modeled through a proliferation probability $p$ and a death probability $d$, which depend on the levels of proliferative and survival potentials of the cells, as well as on the cell micro-environment at time $t$. Importantly, since DTPs and DTEPs exhibit vastly different proliferation rates, we assume that $d$ does not vary with proliferation potential. On the other hand, we assume that maintaining a high survival potential in a drug-free environment is costly to a cell and will act to reduce $p[2,13,14]$. Finally, we assume that $p$ is dependent on the total population size, to represent competition between cells for space and nutrients. This dependence could also be introduced in $d$ without altering the qualitative results of the model.

Stress-induced adaptation of cell proliferation level is modeled by an advection term that leads to a decrease in the level of proliferation. The speed of adaptation $v$ depends on the cell micro-environment at time $t$, the level of survival potential and the average sensitivity of the cell proliferative potential to stress-inducing agents $\bar{v}$.

Finally, we assume that small (large) epimutations correspond to small (large) changes in cell properties, and also that small epimutations occur at a much higher frequency than large epimutations [15]. Therefore, non-genetic phenotype instability is modeled as Brownian motion, or diffusion, in the levels of cell proliferation and survival potentials.

\section{Individual-based model}

In the I-B formalism, we consider each cell as an individual agent, and label it by an index $0 \leq i \leq N(t)$, where $N(t) \geq 0$ is the size of the population at time $t \in[0, T]$, and $T$ is the end time of in silico experiments. The normalized expression levels of the survival-potential and proliferation-potential traits, in each cell $i$ at time $t$, are modeled, respectively, by the random variables $X_{i}(t):[0, \infty) \rightarrow[0,1]$ and $Y_{i}(t):[0, \infty) \rightarrow[0,1]$. We simulate the evolution of the cell population in discrete time, according to the algorithm pictured in Fig.1. Over the time interval between two successive time instants $t$ and $t+\Delta t$, we first allow each cell $i$ either to proliferate, undergo apoptosis or remain in a quiescent state according 
to the respective probabilities

$$
p\left(X_{i}, Y_{i}, N(t)\right) \Delta t, \quad d\left(X_{i}, c(t)\right) \Delta t \quad \text { and } \quad 1-\Delta t\left(p\left(X_{i}, Y_{i}, N(t)\right)+d\left(X_{i}, c(t)\right)\right) .
$$

If a cell proliferates, we assume both daughter cells inherit the parent's trait values. After all cells have undergone one iteration of the proliferation and death process, and returned to the quiescent state, we then let each cell update its trait values according to the following system of discretized Stochastic Differential Equations:

$$
X_{i}(t+\Delta t)=X_{i}(t)+\underbrace{D \sqrt{\Delta t} W_{i}^{1}(t),}_{\begin{array}{c}
\text { non-genetic } \\
\text { instability }
\end{array}} \quad Y_{i}(t+\Delta t)=Y_{i}(t)+\underbrace{D \sqrt{\Delta t} W_{i}^{2}(t)}_{\begin{array}{c}
\text { non-genetic } \\
\text { instability }
\end{array}}+\underbrace{\Delta t v\left(X_{i}(t), c(t) ; \bar{v}\right),}_{\begin{array}{c}
\text { stress-induced adaptation } \\
\text { of the proliferation level }
\end{array}}
$$

where $W_{i}^{1}(t)$ and $W_{i}^{2}(t)$ are standard normal random variables for all $0 \leq i \leq N(t)$ and $t \geq 0$, and $D$ is the average rate of phenotypic fluctuations.

\section{Integro-differential equation model}

In the IDE formalism, we consider the cell population to be structured by two continuous, real variables $x \in[0,1]$ and $y \in[0,1]$, which represent, respectively, the normalized expression levels of survival potential and proliferation potential. The population density of cancer cells is modeled by the function $n(x, y, t) \geq 0$, where the global population density at time $t \in[0, T]$ is computed as $\rho(t)=\int_{0}^{1} \int_{0}^{1} n(x, y, t) \mathrm{d} x \mathrm{~d} y$, and the evolution of $n$ is governed by the following equation:

$$
\frac{\partial n}{\partial t}(x, y, t)+\underbrace{\frac{\partial}{\partial y}(v(x, c(t) ; \bar{v}) n(x, y, t))}_{\begin{array}{c}
\text { stress-induced adaptation } \\
\text { of the proliferation level }
\end{array}}=\underbrace{[p(x, y, \rho(t))-d(x, c(t))] n(x, y, t)}_{\text {selection }}+\underbrace{\frac{D^{2}}{2} \Delta n(x, y, t)}_{\begin{array}{c}
\text { non-genetic } \\
\text { instability }
\end{array}} .
$$

\section{MAIN RESULTS AND PERSPECTIVES}

In [6], we showed that these models are capable of reproducing the main experimental observations detailed in [1]. Our analysis highlighted the important role of non-genetic fluctuations in phenotype in the emergence of drug tolerance in PC9 cancer cell lines. In particular, we suggested that the absence of non-genetic instability can result in the stabilization of the DTP phenotype in the surviving population, so that DTEPs do not emerge, or even in extinction. This is a key result since it supports the idea that epigenetic therapy may be a promising therapeutic strategy in the war against cancer $[16,17,18]$.

Another important prediction of our models is that the transient dominance of DTPs is strictly related to the use of high doses of cytotoxic drugs. If experimentalists apply a lower dose of cytotoxic agents to the PC9 cell population during drug therapy, we propose that it would be highly unlikely to observe DTPs. Rather, we would expect the DTEPs to emerge directly from the PC9 population. Note that this is the usual way to yield stable drug-tolerant lineages [19].

Based on our models, we could conclude that if there are no DTPs present in the initial population of PC9 cells, then it is likely that a proper interplay between non-genetic phenotype instability, stress-induced adaptation and selection is mandatory for the transient appearance of the DTP phenotype during high-dose drug therapy. On the other hand, if there are some DTPs present in the initial population, then non-genetic fluctuations in phenotype and selection are enough to explain the experimental observations reported in [1]. Therefore, the next biologically meaningful question is: are DTPs present in the initial population? Our analysis enabled us to propose a low cytotoxic drug-dose experiment which could answer this question.

In order to keep the models as simple as possible, we chose to include only mechanisms that were necessary to reproduce the experimental results observed in [1]. However, if appropriate future experimental evidence emerges for this system, such as time series data for proliferation rates or cell density, then this could be used to determine whether an additional mechanism, like stress-induced adaptation of the survival potential, should be added to the basic framework established in [6].

A natural way to extend our model would be to study the emergence of epigenetic drug tolerance in a primary tumor. In this framework, we could assume cells to be organized in a non-vascularized and radially symmetric microspheroid [20], and introduce an additional structuring variable that stands for the normalized linear distance of cells 
from the center of the spheroid. We could also introduce an additional evolution equation for the local concentration of cytotoxic drugs. In this case, the interplay between diffusion and consumption of cytotoxic drugs could lead to the creation of distinct niches differentiated by the local environment, which would provide ecological opportunities for diversification [21].

In our study, we have considered a two-dimensional structuring phenotype. It is then natural to wonder about what could be a biological quantitative variable underlying such a continuous phenotype. We propose, as a reasonable candidate, the degree of epigenetic modifications of the DNA (methylation, histone acetylation) in relevant parts of the genome. Such modeling has been previously proposed in [22], using a one-dimensional, phenotype-like structure variable. By using a multidimensional phenotype, we consider differential effects of epimutations on genes responsible for the classical intracellular pathways of proliferation and survival.

\section{ACKNOWLEDGMENTS}

This work was supported in part by the French National Research Agency through the "ANR blanche" project Kibord [ANR-13-BS01-0004]. TL was also supported by the Hadamard Mathematics Labex, backed by the Fondation Mathématique Jacques Hadamard, through a grant overseen by the French National Research Agency [ANR-11LABX-0056-LMH].

\section{REFERENCES}

1. S. V. Sharma, D. Y. Lee, B. Li, M. P. Quinlan, F. Takahashi, S. Maheswaran, U. McDermott, N. Azizian, L. Zou, M. A. Fischbach, et al., Cell 141, 69-80 (2010).

2. C. A. Aktipis, A. M. Boddy, R. A. Gatenby, J. S. Brown, and C. C. Maley, Nat Rev Cancer 13, 883-892 (2013).

3. A. Kreso, C. A. O'Brien, P. van Galen, O. I. Gan, F. Notta, A. M. Brown, K. Ng, J. Ma, E. Wienholds, C. Dunant, et al., Science 339, 543-548 (2013).

4. A. O. Pisco, A. Brock, J. Zhou, A. Moor, M. Mojtahedi, D. Jackson, and S. Huang, Nat Commun 4, 2467 (2013).

5. K. P. Williams, J. L. Allensworth, S. M. Ingram, G. R. Smith, A. J. Aldrich, J. Z. Sexton, and G. R. Devi, Cancer Lett 337, 77-89 (2013).

6. R. H. Chisholm, T. Lorenzi, A. Lorz, A. K. Larsen, L. Neves de Almeida, A. Escargueil, and J. Clairambault, Cancer research 75, 930-939 (2015).

7. A. R. Anderson, A. M. Weaver, P. T. Cummings, and V. Quaranta, Cell 127, 905-915 (2006).

8. D. A. Charlebois, N. Abdennur, and M. Kaern, Phys Rev Lett 107, 218101 (2011).

9. A. S. Silva, and R. A. Gatenby, Biol Direct 5, 25 (2010).

10. M. Delitala, and T. Lorenzi, J Theor Biol 297, 88-102 (2012).

11. O. Lavi, J. M. Greene, D. Levy, and M. M. Gottesman, Cancer Res 73, 7168-7175 (2013).

12. A. Lorz, T. Lorenzi, M. E. Hochberg, J. Clairambault, and B. Perthame, ESAIM-Math Model Num 47, 377-399 (2013).

13. R. J. Gillies, I. Robey, and R. A. Gatenby, J Nucl Med 49, 24S-42S (2008).

14. K. S. Korolev, J. B. Xavier, and J. Gore, Nat Rev Cancer Reviews Cancer 14, 371-380 (2014).

15. C. Becker, J. Hagmann, J. Müller, D. Koenig, O. Stegle, K. Borgwardt, and D. Weigel, Nature 480, 245-249 (2011).

16. T. Clozel, S. Yang, R. L. Elstrom, W. Tam, P. Martin, M. Kormaksson, S. Banerjee, A. Vasanthakumar, B. Culjkovic, D. W. Scott, et al., Cancer Discov 3, 1002-1019 (2013).

17. R. A. Juergens, J. Wrangle, F. P. Vendetti, S. C. Murphy, M. Zhao, B. Coleman, R. Sebree, K. Rodgers, C. M. Hooker, N. Franco, et al., Cancer Discov 1, 598-607 (2011).

18. W. Timp, and A. P. Feinberg, Nat Rev Cancer 13, 497-510 (2013).

19. M. M. Gottesman, Annu Rev Med 53, 615-627 (2002).

20. P. Yu, M. Mustata, L. Peng, J. J. Turek, M. R. Melloch, P. M. French, and D. D. Nolte, Appl Opt 43, $4862-4873$ (2004).

21. A. Lorz, T. Lorenzi, J. Clairambault, A. Escargueil, and B. Perthame, Bulletin of mathematical biology 77, 1-22 (2014).

22. J. Lei, S. A. Levin, and Q. Nie, Proc Natl Acad Sci USA 111, E880-E887 (2014). 